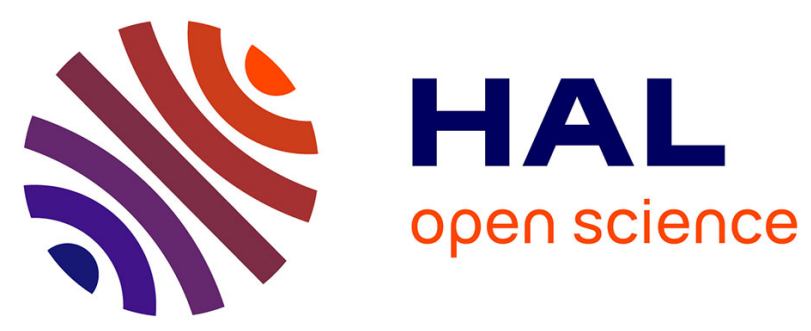

\title{
Analysis of the Delayed Central Nervous System Action in the Regulation of a Third-order Muscle-Tendon Model
}

\author{
Ali El Ati, Islam Boussaada, Silviu-Iulian Niculescu, Pierre-Henry Poret
}

\section{To cite this version:}

Ali El Ati, Islam Boussaada, Silviu-Iulian Niculescu, Pierre-Henry Poret. Analysis of the Delayed Central Nervous System Action in the Regulation of a Third-order Muscle-Tendon Model. ICSTCC 2020 - 24th International Conference on System Theory, Control and Computing, Oct 2020, Sinaia, Romania. hal-02944531

\section{HAL Id: hal-02944531 \\ https://hal.science/hal-02944531}

Submitted on 21 Sep 2020

HAL is a multi-disciplinary open access archive for the deposit and dissemination of scientific research documents, whether they are published or not. The documents may come from teaching and research institutions in France or abroad, or from public or private research centers.
L'archive ouverte pluridisciplinaire HAL, est destinée au dépôt et à la diffusion de documents scientifiques de niveau recherche, publiés ou non, émanant des établissements d'enseignement et de recherche français ou étrangers, des laboratoires publics ou privés. 


\title{
Analysis of the Delayed Central Nervous System Action in the Regulation of a Third-order Muscle-Tendon Model
}

\author{
Ali El Ati ${ }^{\dagger}$, Islam Boussaada* ${ }^{* \dagger}$, Silviu-Iulian Niculescu*,Pierre-Henry Poret ${ }^{\dagger}$ \\ *Université Paris-Saclay, CNRS, CentraleSupélec, Inria \\ Laboratoire des signaux et systèmes (L2S) \\ 91190 Gif-sur-Yvette, France \\ E-mails: \{first name.last name\}@12s.centralesupelec.fr \\ $\dagger$ Institut Polytechnique des Sciences Avancées (IPSA) \\ 63 boulevard de Brandebourg, 94200 Ivry-sur-Seine, France \\ E-mails: \{first name.last name\}@ipsa.fr
}

\begin{abstract}
This paper presents the analysis of a third-order linear time-invariant delay differential equation representing the regulation of a muscle-tendon system. The Central Nervous System action is modeled as a delayed proportionalderivative controller exploiting the multiplicityinduced-dominancy property. The stability analysis is illustrated via the software $\mathbf{P} 3 \delta$.
\end{abstract}

Index Terms - Time-delay systems, stability and stabilization, multiplicity-induced-dominancy, muscle-tendon dynamics, P3 $\delta$ software.

\section{INTRODUCTION}

The control of human motricity is one of the paramount tasks of our Central Nervous System (CNS).To understand the human body movement, one needs not only to establish a good model of the muscle dynamics but also to analyse the control circuit including the structural form of the CNS instruction that controls the dynamic of muscle forces [1]. For instance, abnormal movements caused by neuromuscular diseases, such as Parkinson's disease or sclerosis, result from abnormal muscle tone. Furthermore, maintaining the balance is a vital ability for humans: falls are leading causes of accidental death and morbidity in the elderly, a fact which provides a strong motivation to understand the functioning of the CNS, 2]. Actually, the CNS generates neural commands to activate the muscles. The intern muscles' force combined with inertia and external forces, generate observable movements. The position and velocity of the musculoskeletal system are measured and transmitted to CNS to close the loop with the required information to take appropriate control decisions 3 . However, there is a substantial time-delay caused by

This work is partially supported by a public grant overseen by the French National Research Agency (ANR) as part of the "Investissement d'Avenir" program, through the iCODE project funded by the IDEX Paris-Saclay, ANR-11-IDEX000302. The authors wish to acknowledge the work of the full P3 $\delta$ development team as well as the Cyb'Air Association. the finite speed of signal and the performance of motor tasks is affected by the presence of time-delayed sensory feedback [4], [5]. Moreover, the intrinsic CNS functioning is complex itself, being inherently generator of high-dimensional and nonlinear dynamics. As such, a substantial time delay of signal propagation in the nervous system has to be considered in the input signals. One intuitive and simple way to model such a CNS response is to identify it as a system of propagation which is justified by the necessary lagtime for an information to get through the neuronal axon. We refer the reader to [6] for a summary of the different kinds of delays occurring in neural systems.

The mathematical models that take into account the delay effects in the nervous system are delay functional differential equations which are of infinite dimension and may exhibit a complex dynamical behaviour due to the delay effect. It has been recently shown (see, e.g., 7 - 12$]$ ) that, for some quasipolynomials occurring in systems with time-delays, multiple real roots are often dominant, a property usually referred to as multiplicity-induced-dominancy (MID for short). If, in addition, this multiple dominant root is negative, exponential stability is guaranteed. Namely, a control-oriented MID approach was first proposed in 10 for second-order delay equations then extended in 13. for general $n^{\text {th }}$-order linear time-invariant dynamical systems with a single delay. Indeed, it was shown that under appropriate conditions, the MID property may assess the critical delay established in previous works [9], 14], 15]. In this paper, we consider that the CNS acts as a delayed PD-controller to regulate the muscle dynamics by exploiting the MID property.

Several mathematical models have been proposed to represent muscle dynamics, which are usually classified into three groups 16. The first one is based on black box approximation where the inputs are either the neural signal or the external load, and the output corresponds to either the joint position 
or torque. Hence, the muscle-tendon mechanism may be regarded as a second order system [1]. The second group are Huxley-based distributed-parameter models that seek to explain faithfully the mechanism of contraction with great accuracy, however, its use is not recommended in movement studies because the mathematical complexity of this type of models is paramount and involves ever-increasing numbers of rate functions to describe the chemical and mechanical processes at play in the muscle contraction. Finally, Hill-type lumped-parameter models [17] are the most commonly used to study human movement and postural stability [18], [19], [20]. As a matter of fact, these models describe the mechanical behaviour of the muscle tissue by means of the parallel between the elastic element (PEE), accounting for the passive elastic properties of the muscle fibers, and the contractile element (CE) responsible of the active force generated in the muscle. The elastic properties of the tendon are represented by a spring attached in series with the Hill-type muscle model [21], [22]. Extensive and detailed accounts of Hill-based muscle models are accessible in [16] and [23]. Fig. 1 gives a schematic representation of the muscle-tendon unit using the Hill-type muscle model. In the literature, various linear approximations of Hill models are developed. In particular, the Kelvin-Voight (KV) muscletendon model interprets the mechanical network by the parallel between a single stiffness and a damping element, and the system dynamic is viewed as a second-order equation. It was observed that $\mathrm{KV}$ models present some limits and are particularly not adapted to represent tendon and muscle contractile elements independently [24]. Therefore, a third-order system is proposed in the form of a Poynting-Thomson (PT) model. In this model, a supplementary elastic element is placed in series with a KV model to represent the tendon, bringing them closer to the physiological muscle-tendon system. However, the presence of the tendon element can produce an oscillatory behavior that needs to be compensated by the CNS controller. For the reasons stated above, we shall use a thirdorder PT model to investigate the stability of a muscle-tendon system acting in an arm through the wrist joint.

The paper is organized as follows. Section 2 is dedicated to the modeling of the muscle-tendon interaction as well as to the description of the delayed CNS action. In Section 3, we present the main result where the stability of the muscle response is analysed. Also, the oscillation-inducing critical delay is characterized. Section 4 illustrates the main result by using the recently established P $3 \delta$ software. Section 5 concludes the contribution.

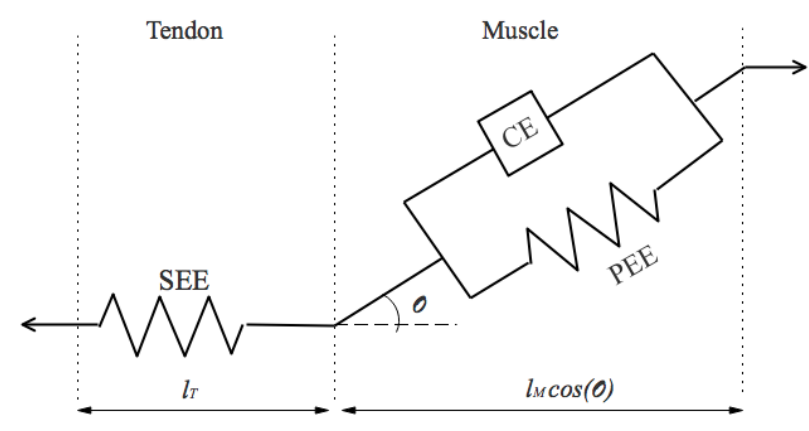

Figure 1. Nomenclature used in the Hill-type muscle model. Each musculotendon actuator is represented by muscle elements in series with an elastic tendon (SEE). The mechanical behavior of muscle is described by a Hill-type contractile element (CE) that models the muscle's force-length-velocity property, and a parallel-elastic element (PEE) that models the muscle's stiffness. (Inspired from [23])

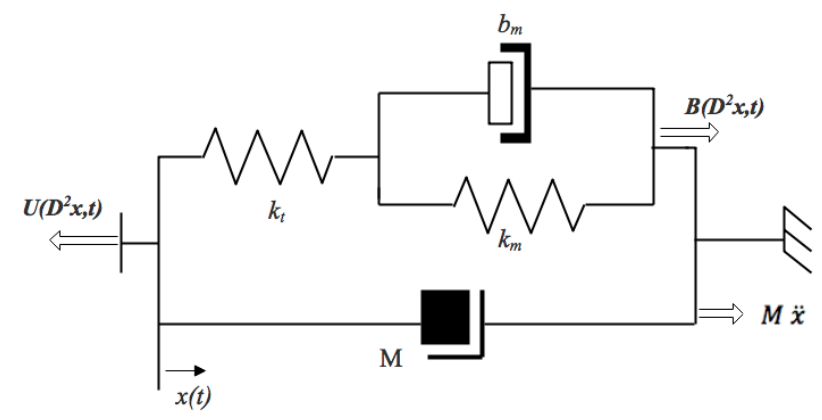

Figure 2. PT model of muscle-tendon system. The mechanical behavior of muscle is described by the parallel between $k_{m}$ and $b_{m}$ that represent the stiffness and viscosity of muscle fibers. The stiffness of the tendon is represented by $k_{t}$. The diagram depicts the different force fields of the D'Alembert equation.(Inspired from 25])

\section{Muscle-tendon Models:}

The Poynting-Thomson (PT) model depicted in Fig. 2 is a linear, time-invariant and third-order system. This mechanical network is an extension of the Kelvin-Voigt model and includes tendon elasticity (Hill-type passive model). The PT model includes two separate elastic elements, the stiffness $k_{t}$ of the tendon in series with the muscle fibers and the parallel between $k_{m}$ and $b_{m}$ represents the stiffness and viscosity of the muscle fibers $\left(b_{m}\right.$ is a contractile element accounting for muscle damping). Most identification techniques proposed in the literature assume the damping and stiffness to be time-invariant. One can show that the interaction of the muscle-tendon model with an inertial system (e.g., the mass of bones and connective tissues) yields a third-order system 24 .

Consider $x(t)$ as a vector of generalized variations of position coordinates (angles, Cartesian co- 
ordinates,...) describing the motion of a mechanical system and define $\mathrm{D}^{n} x$ as the set representing the position coordinates' variations and their derivatives with respect to time up to the $n$-th order so that

$$
\mathrm{D}^{n} x=\left(\frac{\mathrm{d}^{n} x}{\mathrm{dt}^{n}}, \ldots, \frac{\mathrm{d}^{2} x}{\mathrm{dt}^{2}}, \frac{\mathrm{d} x}{\mathrm{dt}}, x\right)
$$

with $n \in \mathbf{N}$.

Owing to the Lagrange-D'Alembert principle, the mechanical system may be written as follows

$$
M(x, t) \frac{\mathrm{d}^{2}}{\mathrm{dt}^{2}}(x(t))+B\left(\mathrm{D}^{2} x, t\right)=U\left(\mathrm{D}^{2} x, t\right)
$$

where $M(x, t)$ is the inertial matrix of the system in the chosen coordinate frame, $B\left(\mathrm{D}^{2} x, t\right)$ is the internal force field generated by the mechanical network, and $U\left(\mathrm{D}^{2} x, t\right)$ is the external force field, (see 24, 25] for more details).

Assuming the system is stationary, classical Laplace transform techniques can be used to recast equation (1) as:

$$
M s^{2} X(s)+B(s)=U(s)
$$

According to the PT model represented in Fig. 2, the transfer function of the internal force generated by the mechanical network is the transfer function of the series of tendon and muscle fibers, namely

$$
\frac{B(s)}{X(s)}=-\frac{k_{t} Z_{m}(s)}{k_{t}+Z_{m}(s)}
$$

where $Z_{m}(s)$ is the transfer function of the muscle fibers composed by the parallel between the stiffness and viscosity of the muscle fibers, which is given by

$$
Z_{m}=b_{m} s+k_{m} \text {. }
$$

Substituting equation (4) in (3) yields the following transfer function

$$
\frac{B(s)}{X(s)}=-\frac{k_{t}\left(b_{m} s+k_{m}\right)}{k_{t}+k_{m}+b_{m} s}
$$

which we rewrite in the following fashion

$B(s)=-\frac{k_{t} b_{m} s}{k_{t}+k_{m}} X(s)-\frac{k_{t} k_{m}}{k_{t}+k_{m}} X(s)-\frac{b_{m}}{k_{t}+k_{m}} s B(s)$

Combining equations (2) and (5), we infer

$$
\left(M s^{2}-\frac{k_{t}\left(b_{m} s+k_{m}\right)}{k_{t}+k_{m}+b_{m} s}\right) X(s)=U(s)
$$

In the absence of external force, it was demonstrated that the PT model with a parameters range in the normal physiological scales often exhibits an oscillatory free response 25]. That is why, the central nervous system (CNS) forces controller is required to cope with such a bio-mechanical constraint by way of a delayed feedback regulation. Hence, we propose that the external force field consists of the CNS action

$\begin{array}{ll}\text { Parameters } & \text { value } \\ k_{t} & 50.1 \mathrm{Nm} / \mathrm{rad} \\ k_{m} & 18.9 \mathrm{Nm} / \mathrm{rad} \\ b_{m} & 0.5 \mathrm{Nms} / \mathrm{rad} \\ \mathrm{M} & 0.002 \mathrm{~kg} / \mathrm{m}^{2}\end{array}$

Table I

NumericAl SETting FOR MUSCLE-TENDON MODEL

$U_{C N S}(s, \tau)$ and an external impulsive perturbation $U_{E X}$, i.e.

$$
U(s)=U_{C N S}(s, \tau)+U_{E X}(s)
$$

Neural feedback control mechanisms for muscle are time-delayed. In other words, there is a significant time interval between the measurement of the variables the application of the forces. Consequently, the CNS input applied to the muscle-tendon system can be represented by

$$
U_{C N S}(s, \tau)=(\alpha+\beta s) e^{-\tau s} X(s)
$$

Then, simple calculations lead to the closed-loop transfer function

$$
F(s)=\frac{X(s)}{U_{E X}(s)}=\frac{k_{t}+k_{m}+b_{m} s}{\tilde{P}(s)+\tilde{Q}(s) e^{-\tau s}},
$$

where

$$
\left\{\begin{aligned}
\tilde{P}(s)= & M b_{m} s^{3}+M\left(k_{t}+k_{m}\right) s^{2}-k_{t} b_{m} s-k_{t} k_{m}, \\
\tilde{Q}(s)= & -\beta b_{m} s^{2}-\left(\alpha b_{m}+\beta\left(k_{t}+k_{m}\right)\right) s \\
& -\left(k_{t}+k_{m}\right) \alpha .
\end{aligned}\right.
$$

\section{Numerical setting :}

We use bio-mechanical properties of muscles and tendon in the arm as reported in the literature (see [5], 25], 26], ). In 25], authors give a good estimation of stiffness parameters for 5 main muscles across both the wrist and elbow. Under the assumption that each muscle contributes to the rotational stiffness of the joint on which it acts, they infer the equivalent joint stiffness at the wrist. Moreover, the arm's moment is calculated where both the wrist and the elbow are completely extended (the joint angle $\theta=0$ ). The numerical parameters of PT muscle-tendon model in the wrist joint are presented in Table 1.

\section{MAIN RESULTS}

We investigate the stabilizing delayed-controller modeling the CNS action, as well as the range of the delay up to the limit of stability, which corresponds to the so-called delay margin. In closed-loop, the corresponding normalized characteristic function reads:

$$
\Delta(s, \tau)=P(s)+Q(s) e^{-\tau s}
$$

where $P$ is a unitary polynomial, i.e, $P=\tilde{P} /\left(M b_{m}\right)$ and $Q=\tilde{Q} /\left(M b_{m}\right)$ 
The main idea of the delayed design based on the MID property is to force the quasipolynomial (12) to have a root of a prescribed multiplicity, bounded by the quasipolynomial degre $\mathrm{T}^{1}$ allowing to tune the controller gains according to a prescribed exponential decay of the closed-loop system solutions. More precisely, the control-oriented MID suggests a root of multiplicity equal to the order of the polynomial associated to the delay plus one. In our case, the CNS acts as a delayed PD-controller.

As a matter of fact, this multiplicity constraint defines a manifold in the parameter space enabling the tuning of the gains $\alpha$ and $\beta$ when the delay $\tau$ is left-free and guaranteeing the exponential stability of the closed-loop system solution. Hereafter, the next theorem, which is based on the MID property, states the explicit conditions on the parameters' values guaranteeing the targeted multiplicity. Recall that the multiplicity of a given root of the generic quasipolynomial 12 is bounded by its degree, so that is 6 is the bound of multiplicity in our case. Introducing the parameters' settings as in Table 1 , the quasipolynomial function 12 becomes:

$$
\begin{aligned}
\Delta(s) & =s^{3}+138 s^{2}-25050 s-946890 \\
& +\left(-500 \beta s^{2}-(69000 \beta+500 \alpha) s-69000\right) \mathrm{e}^{-\tau s}
\end{aligned}
$$

\section{Proposition 1. The following assertions hold:}

i) The multiplicity of a given root of (13) is bounded by 4 .

ii) The only admissible quadruple roots for (13) are in the set $s \in\{-407.6856536,-22.86735033\}$;

iii) The real number $s_{0}=-22.86735033$ is a quadruple root of 13 if, and only if, $\tau_{0}=0.01059835599, \alpha_{0}=-13.75341181, \beta_{0}=$ -0.4143339847 ;

iv) If $s_{0}=-22.86735033$ is a quadruple root of 13 . then $s_{0}$ is also the corresponding spectral abscissa;

v) For a positive delay perturbation $\tau=\tau_{0}+\epsilon$ with $\epsilon>0$, the first crossing frequency $\omega=$ 11.91836147 occurs for $\epsilon=0.0002807867460$.

Proof. The degree of the quasipolynomial function defined in 13 is equal to 6 and the Pólya and Szegö result from [27] asserts that 6 is the generic bound of the multiplicity of any root of $(13)$.

One first investigates the vanishing of the quasipolynomial $\Delta$. It yields the elimination of the exponential term as a rational function in $s$ :

$$
\mathrm{e}^{-\tau s}=\frac{-s^{3}-138 s^{2}+25050 s+946890}{-500 \beta s^{2}+(-69000 \beta-500 \alpha) s-69000 \alpha} .
$$

\footnotetext{
${ }^{1}$ The degree of a given quasipolynomial is defined as the sum of the degrees of the involved polynomials plus the number of corresponding delays.
}

Next, to investigate potential roots with algebraic multiplicity 4 , one substitutes the obtained identity (14) in the ideal $\mathcal{I}_{4}$ generated by the first three derivatives of $\Delta$, i.e., $\mathcal{I}_{4}=<\partial_{s} \Delta, \partial_{s}^{2} \Delta, \partial_{s}^{3} \Delta>$, which yields a manifold defined by three algebraic equations in four unknowns $(s, \alpha, \beta, \tau)$. Solving it and substituting the obtained solution in (14) determines the two admissible solutions given in ii). Furthermore, one easily checks that both of them are not roots of $\partial_{s}^{4} \Delta=0$, which concludes the proof of i).

Items ii-iii) follow directly from the proof of i).

Item iv) follows directly from 13 . Indeed, the main result therein provides sufficient conditions for the dominance of a multiple root, which is essentially based on the real-rootedness of the delay-free polynomial $P$. Namely, one easily proves this fact by computing the discriminant of $P$ the positivity of which guarantees three real roots.

To show item v), the sensitivity analysis with respect to the delay uncertainties is studied, which brings one to investigate the quasipolynomial:

$$
\begin{aligned}
\Delta_{\epsilon}(s)=s^{3} & +138 s^{2}-25050 s-946890 \\
+ & \left(207.1669924 s^{2}+35465.75084 s\right. \\
& +948985.4149) \mathrm{e}^{-(0.01059835599+\epsilon) s}
\end{aligned}
$$

By substituting $s=i \omega$ in the above quasipolynomial, one deduces the corresponding crossing frequencies. Indeed, separating real and imaginary parts, the following system of trigonometric polynomials is satisfied:

$$
\begin{aligned}
& -\left(207.1669924 \omega^{2}-948985.4149\right) \times \\
& \quad \cos (0.01059835599 \omega+\omega \epsilon) \\
& +35465.75084 \omega \sin (0.01059835599 \omega+\omega \epsilon) \\
& -946890-138 \omega^{2}=0 \\
& 35465.75084 \omega \cos (0.01059835599 \omega+\omega \epsilon) \\
& +\left(207.1669924-948985.4149 \omega^{2}\right) \times \\
& \sin (0.01059835599 \omega+\omega \epsilon)-\omega^{3}-25050 \omega=0
\end{aligned}
$$

Eliminating $\cos (0.01059835599 \omega+\omega \epsilon)$ from the first trigonometric polynomial and feeding it into the second, we obtain the frequency $\omega_{c}=11.9183614$. It is then substituted into the expression of $\cos (0.01059835599 \omega+\omega \epsilon)$ to recover the value for $\epsilon_{c}=0.0002807867460$, which concludes the proof of $\mathrm{v})$.

\section{Numerical Simulations Using P3 $\delta$ SOFTWARE}

Based on recent results from [10], [13], [28] on the multiplicity-induced-dominancy property for systems with single time-delays, a Python toolbox for the parametric design of stabilizing feedback laws with time-delays, called Partial Pole Placement via Delay Action (P3 $\delta$ for short), has been developed, see 


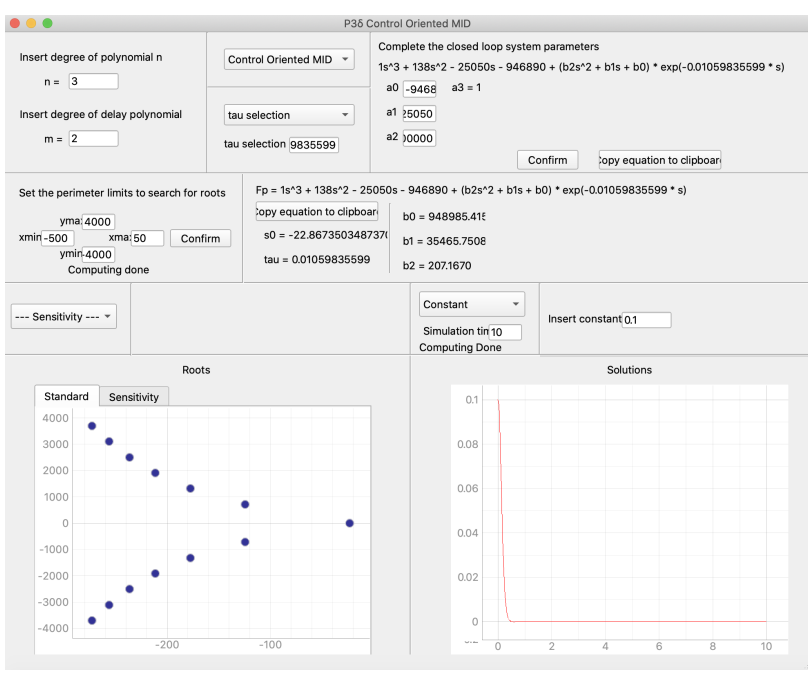

Figure 3. The $\mathrm{P} 3 \delta$ interface exhibiting (left) the spectrum distribution of (13) and (right) the time-domain response of the muscle dynamics in the closed-loop system, in the presence of a quadruple spectral value for 13 at $s=-22.86735033$.

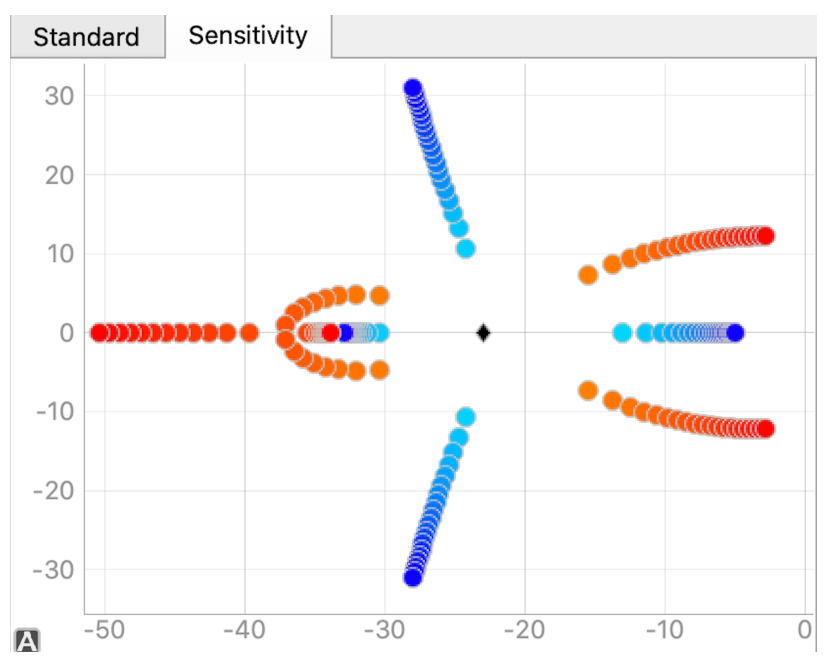

Figure 4. The Sensitivity of the multiple dominant spectral value with respect to delay uncertainty which illustrates item v) of Proposition 1.

[29]. The software is freely available for download on https://cutt.1y/p3delta, where installation instructions, video demonstrations, and a user guide are also available.

In this section, we illustrate the use of $\mathrm{P} 3 \delta$ to numerically investigate the problem we consider; the design of the CNS action as well as the sensitivity of its regulation with respect to the uncertain delay are displayed.

\section{CONCluding REMARKS}

A third-order muscle-tendon model is revisited taking into account the delay in the CNS action. The
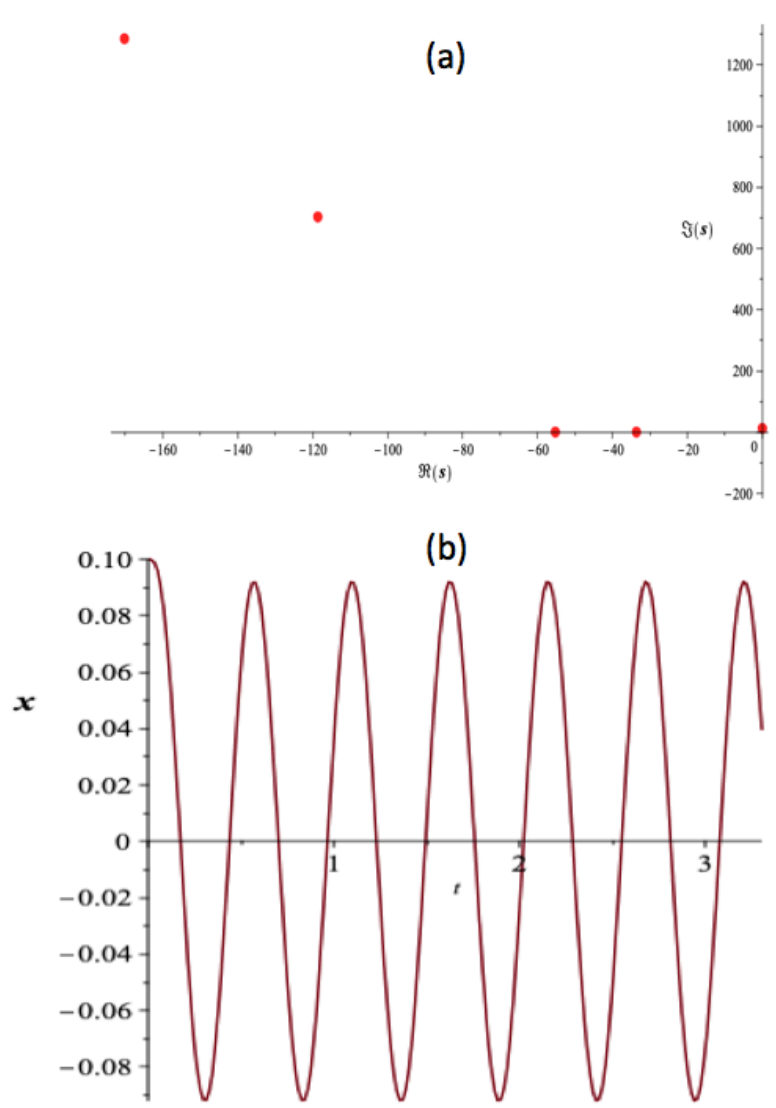

Figure 5. (a) Spectrum distribution for $\tau=0.01087914274$ showing the crossing frequency $\omega=11,91836147 \mathrm{rad} / \mathrm{sec}$. (b) Muscle oscillating response occurring with frequency $\omega=$ $11,91836147 \mathrm{rad} / \mathrm{sec}$ for the delay value $\tau=0.01087914274$.

MID property is exploited to model the CNS action and identify the critical delay inducing oscillations. The P3 $\delta$ software is used to numerically illustrate the qualitative behavior of the muscle dynamics.

\section{REFERENCES}

[1] J. Winters and L. Stark, "Muscle models: What is gained and what is lost by varying model complexity," Biological Cybernetics, vol. 55, pp. 403-420, 1987.

[2] G. Stepan, "Delay effects in the human sensory system during balancing," vol. 367, pp. 1195-1212, 2009.

[3] R. Bortoletto, E. Pagello, and D. Piovesan, "Lower limb stiffness estimation during running: The effect of using kinematic constraints in muscle force optimization algorithms," in Simulation, Modeling, and Programming for Autonomous Robots, D. Brugali, J. Broenink, T. Kroeger, and B. MacDonald, Eds. Springer International Publishing, 2014, pp. 364-375.

[4] J. G. Milton, "The delayed and noisy nervous system: implications for neural control," Journal of Neural Engineering, vol. 8, no. 6, p. 065005, 2011.

[5] R. Begg and M. Palaniswami, Computational Intelligence for Movement Sciences: Neural Networks and Other Emerging Techniques, ser. Computational intelligence and its applications series. Idea Group Pub., 2006.

[6] S. A. Campbell, Time Delays in Neural Systems. Berlin, Heidelberg: Springer Berlin Heidelberg, 2007, pp. 65-90.

[7] I. Boussaada and S.-I. Niculescu, "Tracking the algebraic multiplicity of crossing imaginary roots for generic quasipolynomials: a Vandermonde-based approach," IEEE 
Trans. Automat. Control, vol. 61, no. 6, pp. 1601-1606, 2016.

[8] — "Characterizing the codimension of zero singularities for time-delay systems: a link with Vandermonde and Birkhoff incidence matrices," Acta Appl. Math., vol. 145, pp. 47-88, 2016. [Online]. Available: https://doi.org/10. 1007/s10440-016-0050-9

[9] I. Boussaada, S. Tliba, S.-I. Niculescu, H. U. Ünal, and T. Vyhlídal, "Further remarks on the effect of multiple spectral values on the dynamics of time-delay systems. Application to the control of a mechanical system," Linear Algebra Appl., vol. 542, pp. 589-604, 2018. [Online]. Available: https://doi.org/10.1016/j.laa.2017.11.022

[10] I. Boussaada, S.-I. Niculescu, A. El Ati, R. Pérez-Ramos, and K. Liviu Trabelsi, "Multiplicity-Induced-Dominancy in parametric second-order delay differential equations: Analysis and application in control design," ESAIM: Control, Optimisation and Calculus of Variations, Nov. 2019. [Online]. Available: https://hal.archives-ouvertes.fr/ hal-02421253

[11] G. Mazanti, I. Boussaada, and S.-I. Niculescu, "On qualitative properties of single-delay linear retarded differential equations: Characteristic roots of maximal multiplicity are necessarily dominant," in 21st IFAC World Congress, Berlin, Germany, Jul. 2020

[12] G. Mazanti, I. Boussaada, S.-I. Niculescu, and T. Vyhlídal, in 21st IFAC World Congress, ser. IFAC-PapersOnLine. Berlin, Germany: IFAC, Jul. 2020. [Online]. Available: https://hal.archives-ouvertes.fr/hal-02422707

[13] T. Balogh, T. Insperger, I. Boussaada, and S.-I. Niculescu, "Towards an MID-based Delayed Design for Arbitraryorder Dynamical Systems with a Mechanical Application," in 21st IFAC World Congress, Berlin, Germany, Jul. 2020. [Online]. Available: https://hal.archives-ouvertes.fr/ hal-02889938

[14] I. Boussaada, I.-C. Morarescu, and S.-I. Niculescu, "Inverted pendulum stabilization: Characterization of codimension-three triple zero bifurcation via multiple delayed proportional gains," Systems and Control Letters, vol. 82, pp. 1-9, Aug. 2015. [Online]. Available: https: //hal.archives-ouvertes.fr/hal-01162266

[15] I. Boussaada and S.-I. Niculescu, "On the dominancy of multiple spectral values for time-delay systems with applications," IFAC-PapersOnLine, vol. 51, no. 14, pp. 55-60, 2018.

[16] F. Romero and F. J. Alonso, "A comparison among different hill-type contraction dynamics formulations for muscle force estimation," Mechanical Sciences, vol. 7, no. 1, pp. 19-29, 2016.

[17] A. V. Hill, "The heat of shortening and the dynamic constants of muscle," vol. 126, p. 136?195, 1938.

[18] A. González, A. Cerda-Lugo, A. Cardenas, M. Maya, and D. Piovesan, "A Third-Order Model of Hip and Ankle Joints During Balance Recovery: Modeling and Parameter Estimation," Journal of Computational and Nonlinear Dynamics, vol. 14, no. 10, 2019.

[19] G. S. Sawicki and N. S. Khan, "A simple model to estimate plantarflexor muscle-tendon mechanics and energetics during walking with elastic ankle exoskeletons," IEEE Transactions on Biomedical Engineering, vol. 63, no. 5, pp. 914-923, 2016.

[20] T. DG., "Adjustment of muscle mechanics model parameters to simulate dynamic contractions in older adults," Journal of Biomechanical Engineering, vol. 125, p. 70-77, 2003.

[21] M. M., U. T., Seth, and A. D. SL., "Flexing computational muscle: modeling and simulation of musculotendon dynamics," vol. 135, 2013.

[22] "An optimal control model for maximum-height human jumping," Journal of Biomechanics, vol. 23, no. 12, pp. 1185 - 1198, 1990.

[23] B. Nigg and W. Herzog, Biomechanics of the Musculoskeletal System. WILEY., 2007.

[24] A. Piovesan, D.and Pierobon and F. Mussa-Ivaldi, "Critical damping conditions for third order muscle models: implications for force control," ser. Journal of Biomechanical Engineering, vol. Volume 2: Biomedical and Biotechnology, 2013.
[25] D. Piovesan, A. Pierobon, and F. Mussa-Ivaldi, "Thirdorder muscle models: The role of oscillatory behavior in force control," ser. ASME International Mechanical Engineering Congress and Exposition, vol. Volume 2: Biomedical and Biotechnology, 2012, pp. 493-501.

[26] D. Piovesan, A. Pierobon, P. Dizio, and J. Lackner, "Comparative analysis of methods for estimating arm segment parameters and joint torques from inverse dynamics," vol. 133, 2011.

[27] G. Pólya and G. Szegö, Problems and Theorems in Analysis, Vol. I: Series, Integral Calculus, Theory of Functions. New York, Heidelberg, and Berlin: Springer-Verlag, 1972.

[28] G. Mazanti, I. Boussaada, and S.-I. Niculescu, "Multiplicity-induced-dominancy for delay-differential equations of retarded type," Feb. 2020, working paper or preprint. [Online]. Available: https://hal. archives-ouvertes.fr/hal-02479909

[29] 1. Boussaada, G. Mazanti, S.-I. Niculescu, J. Huynh, F. Sim, and M. Thomas, "Partial pole placement via delay action: A Python software for delayed feedback stabilizing design," in Proceedings of the 24th International Conference on System Theory, Control and Computin, Sinaia, Romania, October 2020. 